\title{
The prognostic value of procalcitonin, C-reactive protein and cholesterol in patients with an infection and multiple organ dysfunction
}

\author{
Siarhei Anatolevich Tachyla, Alexey Viktorovich Marochkov, \\ Artur Leonidovich Lipnitski, and Yulia Gennadevna Nikiforova \\ Department of Anesthesiology and Intensive Care, Mogilev Regional Hospital, Mogilev, Belarus
}

\begin{abstract}
Background: To establish the prognostic value of procalcitonin, C-reactive protein and cholesterol levels for mortality in patients with an infection and multiple organ dysfunction.

Methods: A prospective case-control study was performed, including 67 patients admitted to the intensive care unit with an infection and multiple organ dysfunction in whom cholesterol, procalcitonin, and C-reactive protein levels were measured on admission and during the course of treatment.

Results: The associations between in-hospital mortality and procalcitonin, C-reactive protein, and cholesterol levels were analyzed. Logistic regression analysis showed that cholesterol (odds ratio [OR], 1.858; 95\% CI, 1.170-2.949; P = 0.009) and C-reactive protein (OR, 4.408; 95\% CI, 2.019-9.624; $\mathrm{P}<0.001)$ levels were predictors of mortality. A receiver operating characteristic curve analysis yielded an area under the curve (AUC) of 0.774 and 95\% CI of 0.693-0.855 (P< $0.001)$ for C-reactive protein, an AUC of 0.66 and $95 \%$ CI of $0.535-0.784(\mathrm{P}=0.019)$ for procalcitonin, and an AUC of 0.654 and $95 \%$ CI of $0.593-0.715(\mathrm{P}<0.001)$ for cholesterol as predictors of mortality. When combined with the bioscore system for mortality, these markers yielded an AUC of 0.845 and $95 \%$ CI of $0.770-0.921$ ( $\mathrm{P}<0.001)$, with sensitivity of $89.1 \%$ and specificity of $83.1 \%$.

Conclusions: The combination of procalcitonin, C-reactive protein, and cholesterol levels in a single scoring system yielded high predictive value for mortality.
\end{abstract}

Key Words: Cholesterol, C-reactive protein, Infection, Mortality, Multiple organ dysfunction, Procalcitonin.

Corresponding author: Siarhei Anatolevich Tachyla, M.D.

Department of Anesthesiology and Intensive Care, Mogilev Regional Hospital, 12 Byalynitsky-Birulya Str., Mogilev 212026, Belarus

Tel: 375-222-500881, Fax: 375-222-500373

Email: tsa80@inbox.ru

ORCID: http://orcid.org/0000-0003-1659-5902

Received: September 1, 2016.

Revised: October 20, 2016 (1st); December 6, 2016 (2nd).

Accepted: December 12, 2016.

Korean J Anesthesiol 2017 June 70(3): 305-310

https://doi.org/10.4097/kjae.2017.70.3.305

\section{Introduction}

The search continues for a highly sensitive marker to diagnose sepsis early and predict severity and outcome. Sepsis is lifethreatening organ dysfunction caused by a dysregulated host response to an infection, according to the Third International Consensus Definitions for Sepsis and Septic Shock [1].

Traditional microbiological tests for diagnosing infection are highly specific and easily available in routine practice; however, their sensitivity is only $25-42 \%$, and a negative blood culture result does not guarantee the absence of bacteremia [2,3]. In ad-

(c) This is an open-access article distributed under the terms of the Creative Commons Attribution Non-Commercial License (http://creativecommons.org/ licenses/by-nc/4.0/), which permits unrestricted non-commercial use, distribution, and reproduction in any medium, provided the original work is properly cited. 
dition, $48 \mathrm{~h}$ is needed to obtain the final results, and there is no opportunity to evaluate the contribution of non-culturable microorganisms to the infectious and inflammatory process, which limits the diagnostic ability of the method [2,3].

Clinical and laboratory scales are available to evaluate the severity of multiple organ dysfunction syndrome, such as the Sequential Organ Failure Assessment (SOFA) and the Logistic Organ Dysfunction System [4]. However, scoring only confirms the presence of already developed multiple organ dysfunction. Clinical practice requires markers that predict a high risk of multiple organ dysfunction in patients with an infection.

In recent years, more than 178 biomarkers have been studied and reported in 3,370 articles on the early diagnosis of sepsis [5]. The present study focused on procalcitonin (PCT), C-reactive protein (CRP), and cholesterol as the most accessible and costeffective of these markers.

The objective of this study was to establish the prognostic value of PCT, CRP, and cholesterol levels for mortality in patients with an infection and multiple organ dysfunction.

\section{Materials and Methods}

A prospective case-control study was performed on 67 patients (39 males and 28 females; age, 18-85; mean 58; range, 44-66 years) treated at the intensive care unit (ICU) during 2010-2015.

This study was approved by the Ethics Committee of Mogilev Regional Hospital, and all patients gave written informed consent upon admission to the ICU.

Inclusion criteria were: 1) presence of two or more clinical or laboratory signs of systemic inflammatory response syndrome; 2 ) presence of an established infection site; 3) presence of dysfunction in two or more organ systems: arterial hypoxemia $\left(\mathrm{PaO}_{2} /\right.$ $\mathrm{FIO}_{2}<300 \mathrm{mmHg}$ ); oliguria (urine output $<0.5 \mathrm{ml} / \mathrm{kg} / \mathrm{h}$ for at least $2 \mathrm{~h}$ ); plasma urea $\geq 45 \mathrm{mmol} / \mathrm{L}$; creatinine $>2.0 \mathrm{mg} / \mathrm{dl}$; coagulation abnormalities (International Normalized Ratio >

Table 1. Patient Demographics in the Study Population

\begin{tabular}{lccl}
\hline & $\begin{array}{c}\text { Survivors } \\
(\mathrm{n}=38)\end{array}$ & $\begin{array}{c}\text { Non-survivors } \\
(\mathrm{n}=29)\end{array}$ & $\mathrm{P}$ \\
\hline Age (yr) & $46.0(40-61)$ & $62.5(56-75)$ & 0.007 \\
Sex (M/F) & $22 / 16$ & $17 / 12$ & 0.95 \\
Body weight (kg) & $81.5(73-88)$ & $79.5(74-89)$ & 0.98 \\
Height (cm) & $170.0(164-176)$ & $170.0(170-176)$ & 0.59 \\
APACHE III (points) & $34.6(19.2-57.4)$ & $65.5(46.7-84.7)$ & 0.01 \\
SOFA (points) & $6.0(4-7)$ & $11.0(8-11)$ & 0.005 \\
\hline
\end{tabular}

Values are expressed as median and quartiles. APACHE: Acute Physiology and Chronic Health Evaluation, SOFA: Sequential Organ Failure Assessment.
1.5 or activated partial thromboplastin time $>60$ s; ileus; thrombocytopenia $<100,000$ platelets $/ \mu \mathrm{l}$ ); inadequate tissue perfusion (lactic acidemia $>2 \mathrm{mmol} / \mathrm{L}$ ); hyperbilirubinemia (plasma total bilirubin $>70 \mathrm{mmol} / \mathrm{L}$ ); altered mental status; arterial hypotension (systolic blood pressure $<90 \mathrm{mmHg}$ or mean arterial pressure $<70 \mathrm{mmHg}$ ); 4) either sex; and 5) aged $>18$ years.

The exclusion criteria were: 1) chronic cardiovascular or decompensated respiratory failure; 2) stage 5 chronic kidney disease and undergoing hemodialysis; 3) hepatic cirrhosis or chronic liver failure; 4) immunodeficiency; 5) glucocorticoid treatment; 6) hypolipidemic treatment; 7) AIDS; 8) metastatic cancer; 9) lymphoma; 10) leukemia; or 11) multiple myeloma.

The patient demographics are presented in Table 1.

The underlying medical conditions of the patients are listed in Table 2. The immediate reason for hospitalization was the development of organ dysfunction that required hemodynamic support with vasoactive and inotropic agents, or mechanical ventilation, among others. These medical conditions occurred within 4-22 days after disease onset (mean, 7.2 \pm 5.1 days). The patients received treatment in accordance with protocols adopted in the Republic of Belarus for each individual nosology; additional support of organs and systems was provided depending on the dysfunction.

Pathological flora was isolated from biological media during bacteriological studies in all patients; from sputum in 55 patients (81.1\%), from urine in 42 patients (62.7\%), from blood in 39 patients (58.2\%), from wound exudate in 24 patients (35.8\%), from drainage tube discharge in 22 patients (32.8\%), from liquor in four patients $(6.0 \%)$, and from the catheter tip in three patients (4.5\%).

PCT, CRP, and cholesterol levels were determined in all patients on admission and during the treatment course. Blood sampling was performed from 8:00 to 9:00 AM, on an empty stomach, and from a peripheral vein not used for infusion therapy. Intravenous fat emulsions were discontinued before and during blood sampling. Whole blood was delivered to the laboratory where the analysis was carried out within $1 \mathrm{~h}$. Cholesterol and CRP levels were determined using the AU 680 biochemi-

Table 2. Underlying Medical Conditions in the Study Population

\begin{tabular}{lcc}
\hline \multicolumn{1}{c}{ Disease } & $\begin{array}{c}\text { Survivors } \\
(\mathrm{n}=38)\end{array}$ & $\begin{array}{c}\text { Non-survivors } \\
(\mathrm{n}=29)\end{array}$ \\
\hline Peritonitis & $9(23.7 \%)$ & $10(34.5 \%)$ \\
Pancreatitis & $7(18.4 \%)$ & $8(27.6 \%)$ \\
Urosepsis & $9(23.7 \%)$ & $6(20.7 \%)$ \\
Pneumonia & $7(18.4 \%)$ & $5(17.2 \%)$ \\
Purulent meningoencephalitis & $4(10.5 \%)$ & 0 \\
Osteomyelitis & $2(5.3 \%)$ & 0 \\
\hline
\end{tabular}

Values are expressed as quantity and percentages. 
cal analyzer (Beckman Coulter, Brea, CA, USA). Enzymatic and immunoturbidimetric methods were used to determine cholesterol and CRP levels, respectively. Before sampling, the device was calibrated using the system calibrator sets and quality control was performed using reference sera. PCT levels were determined using the EasyReader immunochromatographic rapid test analyzer (Vedalab, Cerisé, France) and a set of PROCALCHECK-1 reagents were used for calibration.

Compliance with the normal distribution was determined by the Shapiro-Wilks test. Differences between the independent and dependent variables between the groups were determined using the Mann-Whitney and Wilcoxon tests, respectively. To assess the degree of dependence between the variables, Spearman's correlation coefficient $\mathrm{R}$ analysis was applied. The associations between the markers and mortality as a dependent variable were assessed by logistic regression analysis. A receiver operating characteristic (ROC) curve was plotted and the area under the ROC curve (AUC) was assessed as well as sensitivity and specificity to evaluate the discriminatory power of the markers for predicting mortality. The bioscore system for mortality was analyzed for the AUC, and the subsequent ROC curves were used to evaluate prognostic value. The Youden index was applied to set the cut-offs and was compared between the evaluation methods.

Data are presented as mean and SD (normal distribution), median and quartiles (non-normal distribution), or as percentages for categorical variables. The Statistica 7.0 (Statsoft Inc., Tulsa, OK, USA) and SPSS (SPSS Inc., Chicago, IL, USA) software packages were used for analyses. Differences were considered significant at $\mathrm{P}<0.05$.

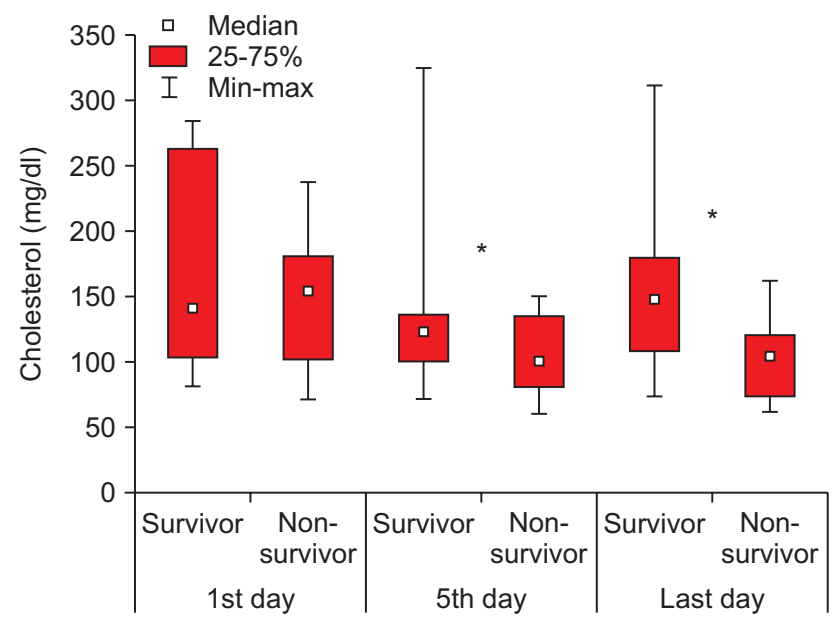

Fig. 1. Cholesterol levels in the two groups. *Significant differences between cholesterol levels in the first and second groups $(\mathrm{P}<0.05)$.

\section{Results}

The patients were divided into two groups, depending on disease outcome. The first group included patients with a positive response to the treatment and recovery $(n=38)$, and the second group included patients who did not respond to treatment and died $(n=29)$. Patients in the two groups did not differ in sex, body weight, or height (Table 1). Patients in the second group were significantly older and had higher Acute Physiology and Chronic Health Evaluation III and SOFA scores on admission.

Several stages were analyzed, depending on the duration of the patient's stay in the ICU. The last day of ICU treatment in the first group - was the last day before transfer out of the ICU, and that in the second group - was the day before death.

No difference in cholesterol level was observed between the groups on day 1 of ICU treatment (Fig. 1; P > 0.05). However, cholesterol levels decreased significantly in both groups on day 5 ; decreases from $174.2(102.6-262.8) \mathrm{mg} / \mathrm{dl}$ to $148.6(117.3-170.2)$ $\mathrm{mg} / \mathrm{dl}(\mathrm{P}=0.03)$ and from $144.4(102.2-180.0) \mathrm{mg} / \mathrm{dl}$ to 116.1 (102.1-227.4) mg/dl $(\mathrm{P}=0.01)$ were observed in the first and second groups, respectively. Cholesterol level was significantly higher in the first group than that the second group $(\mathrm{P}=0.035)$. On the last day of ICU treatment, the cholesterol level in the first group did not change and was 164.1 (104.1-189.6) mg/dl, whereas that in the second group decreased further from the baseline to $100.6(73.5-120.7) \mathrm{mg} / \mathrm{dl}(\mathrm{P}=0.0028)$. In addition, cholesterol level was significantly lower in the second group than that of the first group on the last day of treatment $(\mathrm{P}=0.016)$.

Spearman's correlation coefficient $\mathrm{R}$ for the comparison between cholesterol and PCT levels ranged from -0.19 to -0.61 at different stages and ranged from -0.22 to -0.58 when comparing cholesterol and CRP levels at different stages. PCT and CRP levels were correlated $(\mathrm{R}=0.44-0.63)$ at different stages.

The associations between in-hospital mortality and PCT, CRP, and cholesterol levels were analyzed. A logistic regression analysis showed a significant association with cholesterol level as a predictor of mortality (odds ratio [OR], 1.858; 95\% CI, 1.170-2.949; $\mathrm{P}=0.009$ ), and CRP level (OR, 4.408; 95\% CI, 2.019-9.624; $\mathrm{P}<0.001)$. No association was found between PCT level and mortality (OR, 2.571; 95\% CI, 0.591-11.198; P = 0.208). However, a significant association was found between PCT level at certain stages beginning on day 12 until the last day (OR, 28.0; 95\% CI, 2.067-379.265; P =0.012). The value of CRP as a predictor of mortality also increased beginning on day 13 to the last day (OR, 24.0; 95\% CI, 2.783-206.969; P = 0.004). No such association was observed for cholesterol level.

The ROC curve to assess PCT level as a predictor of mortality is shown in Fig. 2.

The AUC for PCT was 0.66 at a $95 \%$ CI of $0.535-0.784$ (P = 0.019 ) with sensitivity of $88.2 \%$ and specificity of $43.6 \%$ (Youden 


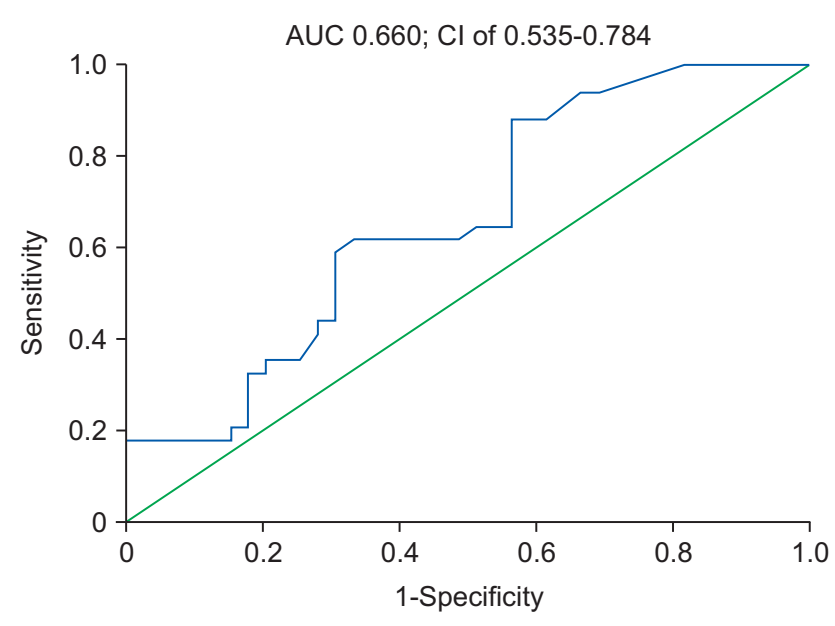

Fig. 2. Receiver operating characteristic curve for procalcitonin. AUC: area under the curve.

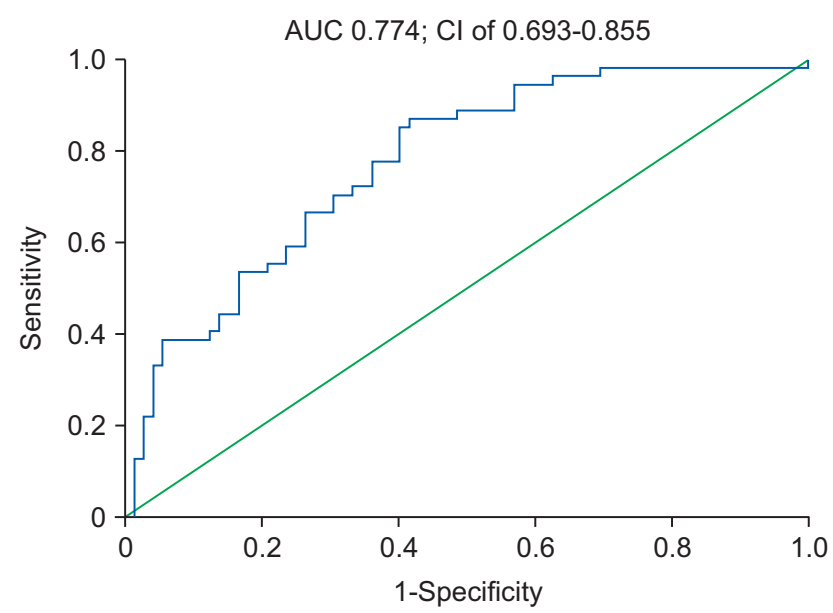

Fig. 3. Receiver operating characteristic curve for C-reactive protein. AUC: area under the curve.

index $\mathrm{J}=0.318$ ). The threshold level for PCT as a predictor of mortality from this ROC curve was $0.8 \mu \mathrm{g} / \mathrm{L}$.

The ROC curve to assess CRP level as a predictor of mortality is shown in Fig. 3.

The AUC for CRP was 0.774 at a $95 \%$ CI of $0.693-0.855(\mathrm{P}<$ 0.001 ), with sensitivity of $87.0 \%$ and specificity of $58.3 \%$ (Youden index $J=0.454$ ). The threshold level for CRP as a predictor of mortality from this ROC curve was $90 \mathrm{mg} / \mathrm{L}$.

The ROC curve to assess cholesterol level as a predictor of mortality is shown in Fig. 4.

The AUC for cholesterol was 0.654 at a $95 \%$ CI of $0.593-0.715$ $(\mathrm{P}<0.001)$, with sensitivity of $82.5 \%$ and specificity of $40.7 \%$ (Youden index $\mathrm{J}=0.262$ ). The threshold level for cholesterol as a predictor of mortality from this ROC curve was $100 \mathrm{mg} / \mathrm{dl}$.

We developed a bioscore system for mortality, which includes

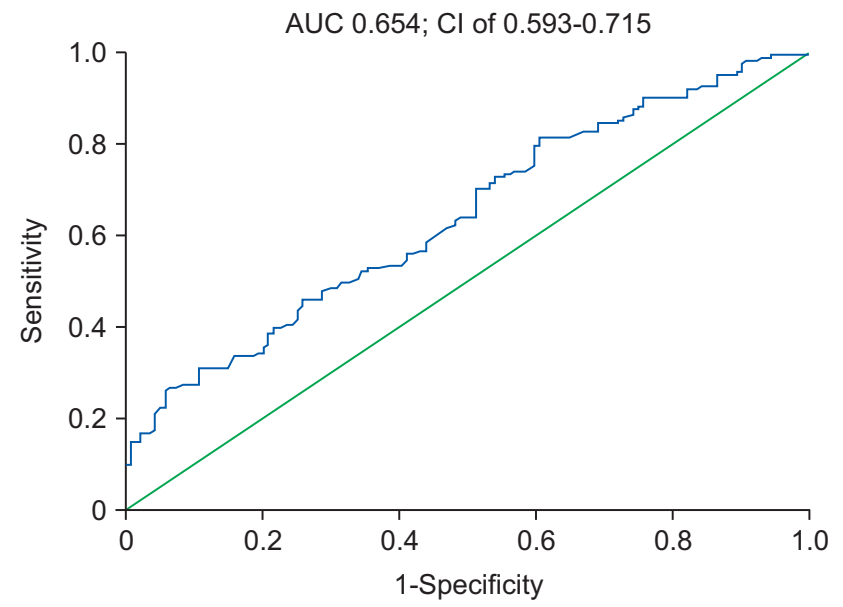

Fig. 4. Receiver operating characteristic curve for cholesterol. AUC: area under the curve.

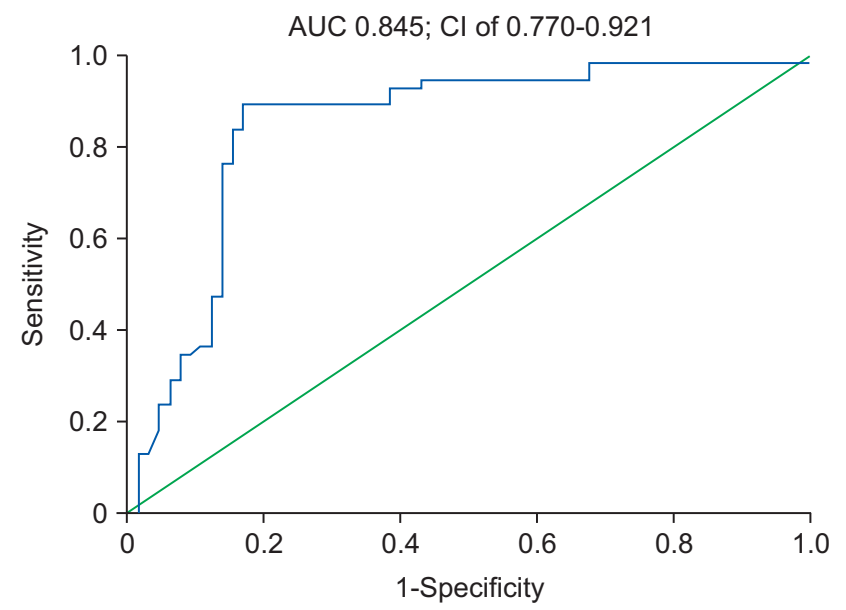

Fig. 5. Receiver operating characteristic curve for the combined bioscore system for mortality in corporating procalcitonin, C-reactive protein and cholesteral. AUC: area under the curve.

simultaneous determination of PCT, CRP, and cholesterol levels as predictors of mortality in patients with infection and multiple organ dysfunction. The resulting ROC curve is shown in Fig. 5.

When combined in a single score system, the markers yielded an AUC of 0.845 at a $95 \%$ CI of $0.770-0.921(\mathrm{P}<0.001)$ with sensitivity of $89.1 \%$ and specificity of $83.1 \%$ (Youden index $\mathrm{J}=$ $0.722)$.

\section{Discussion}

PCT and CRP are commonly used well-established markers for sepsis diagnosis, but they have a number of limitations. PCT level also increases in response to non-bacterial inflammatory conditions, such as polytrauma, major surgery, and persistent cardiogenic shock [6,7]. Increased CRP level can be observed in 
local infections and in non-infectious inflammatory processes (e.g., autoimmune diseases, and malignant tumors) and during the postoperative period and may last for a few days after the suppurative focus sanitation [6,7].

Hypocholesterolemia is commonly seen in patients with severe acute infection $[8,9]$. Although a decrease in serum cholesterol levels were discovered in patients with an infection more than 100 years ago, the association between cholesterol level and critical condition of a patient has not been conclusively established.

In this study, patients with an infection and multiple organ dysfunction showed reduced cholesterol levels on day 5 after admission to the ICU. Such a reduction was also observed in high- and low-density lipoproteins [10,11]. Similar results were obtained by other authors. Van Leeuwen et al. [10] reported decreases in total cholesterol and lipoprotein concentrations by $50 \%$ in patients with severe sepsis on day 3 of observation and a slow increase to baseline by day 28. Chenaud et al. [12] showed that decreasing serum apolipoprotein AI levels are correlated with increasing levels of sepsis markers, such as CRP, PCT, and interleukin-6. The critical cholesterol levels in these patients were $100-135 \mathrm{mg} / \mathrm{dl}$, depending on the study [13-15]. In the present study, the threshold level of cholesterol was $100 \mathrm{mg} / \mathrm{dl}$.

Several studies have demonstrated that hypocholesterolemia in patients with sepsis is a predictor of increased mortality $[8,9,16]$. Our results are comparable with the results obtained by a number of authors. Biller et al. [17] found that a cholesterol level $>80 \mathrm{mg} / \mathrm{dl}$ is associated with a survival AUC of 0.715 in patients with infection, with sensitivity of $72 \%$ and specificity of $66 \%$. Kitazawa et al. [15] reported that patients with a bloodstream infection and lower cholesterol levels tended to have higher 30 day mortality, with an AUC of 0.66 , sensitivity of $100 \%$ and specificity of $39 \%$.

Few comparative studies have determined the sensitivity and specificity of PCT, CRP, and cholesterol levels as prognostic factors for disease outcome. Available studies have presented conflicting results. Biller et al. [17] showed that CRP and PCT levels do not predict mortality (CRP and PCT AUCs of 0.407 and 0.474, respectively). Zhou and Ho [18] found that CRP > $100 \mathrm{mg} / \mathrm{L}$ and PCT $>0.6 \mu \mathrm{g} / \mathrm{L}$ at ICU discharge were associated with an increased risk for adverse post-ICU events. However, PCT yielded an AUC of 0.61 (95\% CI, 0.55-0.66) and CRP con- centration $>100 \mathrm{mg} / \mathrm{L}$ was associated with an increased risk for adverse post-ICU events in a multivariate analysis. Huang et al. [19] showed that dynamic changes in PCT 48 h (day 3) and 96 $\mathrm{h}$ (day 5 ) after admission to the ICU predict survival in patients with severe sepsis and septic shock. Two studies analyzed CRP concentration as a predictor of in-hospital mortality after ICU discharge [20,21]. The AUCs were 0.85 (95\% CI, 0.73-0.96) and 0.87 (95\% CI, 0.73-0.99), respectively.

Some results differ from those described by Biller et al. [17], but are similar to those obtained by Huang et al. [19], Ho et al. [20], and Litton et al. [21]. The use of different intensive therapy protocols for severe infections, initiating early treatment during the period between the onset of infection and the administration of effective antibacterial therapy, source control, and damage to specific target organs in patients with multiple organ dysfunction may affect mortality and alter the statistical significance of biomarkers. This issue requires further, more thorough studies.

The use of combinations of several markers has been considered for the diagnosis of sepsis. Yang et al. [22] suggested a combination of CRP, PCT and sepsis-related organ failure score for diagnosing sepsis in critical patients. The individual values were as follows: CRP (AUC, 0.729; 95\% CI, 0.671-0.787), PCT (AUC, 0.711 ; 95\% CI, 0.652-0.770) and SOFA (AUC, 0.670; 95\% CI $0.607-0.733)$. Combining these values into the bioscore resulted in an AUC of 0.790 (95\% CI, 0.739-0.834).

In conclusion, 1) Cholesterol, PCT, and CRP, can be used as predictors of mortality in patients with an infection and multiple organ dysfunction. A decrease of $<100 \mathrm{mg} / \mathrm{dl}$ in cholesteral level is an unfavorable sign in terms of disease outcome prediction. Determining the cholesterol concentration several times during the course of treatment is more informative than a single determination. 2) The diagnostic values of cholesterol, PCT, and CRP as predictors of mortality were similar, with medium prognostic efficiency. Treatment is recommended at the same time as defining these three criteria to improve their diagnostic value. 3) A weak negative correlation was detected between the cholesterol level and CRP and PCT levels. These markers probably reflect different processes. Increasing CRP and PCT levels indicate the development of a systemic inflammatory response, whereas a decreasing cholesterol level represents a metabolic disorder associated with the development of multiple organ dysfunction.

\section{References}

1. Singer M, Deutschman CS, Seymour CW, Shankar-Hari M, Annane D, Bauer M, et al. The Third International Consensus Definitions for Sepsis and Septic Shock (Sepsis-3). JAMA 2016; 315: 801-10.

2. Mancini N, Burioni R, Clementi M. Microbiological diagnosis of sepsis: the confounding effects of a "gold standard". Methods Mol Biol 2015; 1237: 1-4. 
3. Andrade SS, Bispo PJ, Gales AC. Advances in the microbiological diagnosis of sepsis. Shock 2008; 30 Suppl 1: 41-6.

4. Luo XP, Wang HH, Hu SF, Wu SJ, Xie GH, Cheng BL, et al. Comparison of three different organ failure assessment score systems in predicting outcome of severe sepsis. Zhonghua Wai Ke Za Zhi 2009; 47: 48-50.

5. Liu B, Chen YX, Yin Q, Zhao YZ, Li CS. Diagnostic value and prognostic evaluation of Presepsin for sepsis in an emergency department. Crit Care 2013; 17: R244.

6. Ruiz-Alvarez MJ, García-Valdecasas S, De Pablo R, Sanchez García M, Coca C, Groeneveld TW, et al. Diagnostic efficacy and prognostic value of serum procalcitonin concentration in patients with suspected sepsis. J Intensive Care Med 2009; 24: 63-71.

7. Luzzani A, Polati E, Dorizzi R, Rungatscher A, Pavan R, Merlini A. Comparison of procalcitonin and C-reactive protein as markers of sepsis. Crit Care Med 2003; 31: 1737-41.

8. Leardi S, Altilia F, Delmonaco S, Cianca G, Pietroletti R, Simi M. Blood levels of cholesterol and postoperative septic complications. Ann Ital Chir 2000; 71: 233-7.

9. Fraunberger P, Nagel D, Walli AK, Seidel D. Serum cholesterol and mortality in patients with multiple organ failure. Crit Care Med 2000; 28: 3574-5.

10. van Leeuwen HJ, Heezius EC, Dallinga GM, van Strijp JA, Verhoef J, van Kessel KP. Lipoprotein metabolism in patients with severe sepsis. Crit Care Med 2003; 31: 1359-66.

11. Wendel M, Paul R, Heller AR. Lipoproteins in inflammation and sepsis. II. Clinical aspects. Intensive Care Med 2007; 33: 25-35.

12. Chenaud C, Merlani PG, Roux-Lombard P, Burger D, Harbarth S, Luyasu S, et al. Low apolipoprotein A-I level at intensive care unit admission and systemic inflammatory response syndrome exacerbation. Crit Care Med 2004; 32: 632-7.

13. Marik PE. Dyslipidemia in the critically ill. Crit Care Clin 2006; 22: 151-9.

14. Chiarla C, Giovannini I, Giuliante F, Vellone M, Ardito F, Nuzzo G. Hypocholesterolemia in critical illness. Crit Care Med 2009; 37: 2681-2.

15. Kitazawa T, Yanagimoto S, Tatsuno K, Fukushima A, Okugawa S, Ota Y. Serum cholesterol levels at the onset of bloodstream infection have prognostic value. Adv Infect Dis 2012; 2: 100-5.

16. Guo Q, Zou G. Progress of anti-infection of high density lipoprotein. Zhong Nan Da Xue Xue Bao Yi Xue Ban 2013; 38 : 954-8.

17. Biller K, Fae P, Germann R, Drexel H, Walli AK, Fraunberger P. Cholesterol rather than procalcitonin or C-reactive protein predicts mortality in patients with infection. Shock 2014; 42: 129-32.

18. Zhou G, Ho KM. Procalcitonin concentrations as a predictor of unexpected readmission and mortality after intensive care unit discharge: a retrospective cohort study. J Crit Care 2016; 33: 240-4.

19. Huang MY, Chen CY, Chien JH, Wu KH, Chang YJ, Wu KH, et al. Serum procalcitonin and procalcitonin clearance as a prognostic biomarker in patients with severe sepsis and septic shock. Biomed Res Int 2016; 2016: 1758501.

20. Ho KM, Lee KY, Dobb GJ, Webb SA. C-reactive protein concentration as a predictor of in-hospital mortality after ICU discharge: a prospective cohort study. Intensive Care Med 2008; 34: 481-7.

21. Litton E, Ho KM, Chamberlain J, Dobb GJ, Webb SA. C-reactive protein concentration as a predictor of in-hospital mortality after ICU discharge: a nested case-control study. Crit Care Resusc 2007; 9: 19-25.

22. Yang Y, Xie J, Guo F, Longhini F, Gao Z, Huang Y, et al. Combination of C-reactive protein, procalcitonin and sepsis-related organ failure score for the diagnosis of sepsis in critical patients. Ann Intensive Care 2016; 6: 51. 